\title{
Wannier-Stark Ladders in Driven Optical Lattices
}

\author{
M. Glück, M. Hankel, A. R. Kolovsky[*] and H. J. Korsch \\ Fachbereich Physik, Universität Kaiserslautern, D-67653 Kaiserslautern, Germany
}

January 11,2000

\begin{abstract}
The paper studies the effect of a weak periodic driving on metastable Wannier-Stark states. The decay rate of the ground Wannier-Stark states as a continuous function of the driving frequency is calculated numerically. The theoretical results are compared with experimental data of Ref. [8] obtained for cold sodium atoms in an accelerated optical lattice.

PACS. 0 3.75.-b, 42.50.Hz, 03.65.-w, 73.20.Dx
\end{abstract}

1. The problem of quantum states of a particle in a periodic potential subject to a homogeneous field belongs to one of the most debated problems of one-particle quantum mechanics. It took more than 30 years to realize that these states are resonances arranged in a regular way (the so-called Wannier-Stark ladder of resonances). The contributions by Bloch [1], Landau and Zener [2], and Wannier [3] (followed by a vivid discussion with Zak [4]) are the milestones of the early studies. This problem has not lost its actuality till now and there is a permanent rate of publications on the subject in the literature. In particular we note a recent paper [5], where the Wannier-Stark resonances are introduced rigorously as complex poles of some efficient scattering matrix.

Along with the theoretical analysis the Wannier resonances were studied experimentally. The first indication of Wannier-Stark ladders was found in an experiment on optical absorption in a solid crystal [6]. The next experimental object were superlattices in a semiconductor [7]. Unfortunately, solid state systems do not offer a direct testing of the theory because of the presence of relaxation processes (scattering by impurities, lattice vibrations) and the Coulomb interaction. Recently, the Wannier-Stark ladder was observed in an experiment with cold neutral atoms in a standing laser wave [8]. This system suggests a onedimensional realization of a solid state system

$$
\widehat{H}=\frac{\hat{p}^{2}}{2 M}+V_{0} \cos \left(2 k_{L} x\right)+\operatorname{Max},
$$

which is practically free from relaxation processes and multiparticle interactions. (In the Hamiltonian (1), $M$ is the atomic mass, $k_{L}$ the wave number of the laser, $V_{0}$ the amplitude of the optical potential proportional to the laser intensity, and $M a$ the inertial force due to the acceleration of the standing wave.) In the experiment [8] an additional time-periodic perturbation was imposed,

$$
V_{0} \cos \left(2 k_{L} x\right) \longrightarrow V_{0} \cos \left[2 k_{L} x-\epsilon \cos (\omega t)\right],
$$

and the survival probability $P_{t}(\omega)=\exp [-t / \tau(\omega)]$ for the ground Wannier states was measured as a function of the driving frequency $\omega$. The observed $\omega$-dependence of $P_{t}(\omega)$ shows a pronounced fluctuation which was interpreted as an indication of the Wannier-Stark ladder. In this paper we study the induced (by periodic driving) decay of the ground Wannier-Stark states theoretically. We confirm the relation of the observed structure of $P_{t}(\omega)$ to the WannierStark ladder and analyze the functional dependence of the lifetime $\tau(\omega)$ on the system parameters.

2. The simplest approach to the problem (which is mostly in line with the arguments of Ref. [8]) uses the Fermi golden rule [9]. In fact, it is well known that quantum resonances can be alternatively considered as a peculiarity in the density of state of a system with a continuous spectrum $\rho_{\text {res }}(E) \sim \Gamma_{\text {res }} /\left[\left(E-E_{\text {res }}\right)^{2}+\Gamma_{\text {res }}^{2} / 4\right]$, where $E_{\text {res }}$ and $\Gamma_{\text {res }}$ are the resonance position and the resonance width, respectively. Let us assume now that initially only the ground resonances are populated (which is the case realized in the experiment). The width $\Gamma_{0}$ of the ground resonances is very small and can be set to zero. Then the "initial state" is a ladder of discrete levels

$$
E_{l}=E_{0}+l \hbar \omega_{B}, \quad l=0, \pm 1, \pm 2, \ldots
$$

where $\omega_{B}=\pi M a / \hbar k_{L}$ is the Bloch frequency and $E_{0}$ is the position of the ground resonances reduced to the fundamental energy interval $0 \leq E<\hbar \omega_{B}$. The periodic perturbation lifts the atoms from this "initial state" to the "final state", where they rapidly decay (i.e. become accelerated by the static field). The "final state" is a superposition of all resonances belonging to the excited Wannier-Stark ladders. (In what follows we shall label the different Wannier-Stark ladders by an index $\alpha$.)

For the system parameters used in the experiment cited only the resonances belonging to the first excited $(\alpha=1)$ Wannier-Stark ladder are of importance. The widths $\Gamma_{\alpha}$ of higher resonances $(\alpha>1)$ are very large and contribute to 
the smooth background spectrum $\rho_{b}(E)$. Thus, the "final state" has a density of states

$$
\rho(E)=\rho_{b}(E)+\sum_{l=-\infty}^{\infty} \frac{\Gamma_{1} / 2}{\left(E-E_{1}-l \hbar \omega_{B}\right)^{2}+\Gamma_{1}^{2} / 4},
$$

where $E_{1}$ is the position of the first excited resonances reduced to the energy interval $0 \leq E<\hbar \omega_{B}$. According to the Fermi rule the transition rate is given by the product of the square of the transition matrix element $d\left(E_{0} \rightarrow E\right)$ (which is proportional to the driving amplitude $\epsilon$ ) and the density of states in the continuum. Thus the induced decay rate for the ground states is

$$
\tau^{-1}(\omega)=\tau_{0}^{-1}+\hbar^{-1}|d(\hbar \omega)|^{2} \rho\left(E_{0}+\hbar \omega\right),
$$

where the term $\tau_{0}^{-1}=\Gamma_{0} / \hbar$ takes into account a finite lifetime of the ground states in the absence of the ac field due to Landau-Zener tunneling. Assuming a smooth $\omega$ dependence of the matrix element $d(\hbar \omega)$ we can approximate Eq. (5) by the equation

$\tau^{-1}(\omega)=\tau_{0}^{-1}+\sum_{l} \frac{\left|d_{l}\right|^{2}}{\hbar} \frac{\Gamma_{1} / 2}{\left(\hbar \omega+E_{0}-E_{1}-l \hbar \omega_{B}\right)^{2}+\Gamma_{1}^{2} / 4}$

where $d_{l}=d\left(E_{1}-E_{0}+l \hbar \omega_{B}\right)$. It follows from Eq. (6) that the inverse lifetime of the ground Wannier-Stark states (as a function of driving frequency $\omega$ ) should exhibit a number of equidistant peaks of the same width $\Gamma_{1}$ given by the width of the first excited Wannier-Stark state.

3. The phenomenological approach described above captures, in its best, only the main features of $\tau(\omega)$. To get more precise predictions we employ here a different approach based on the notion of quasienergies. In fact, the relevant states of a quantum system affected by a timeperiodic perturbation are quasienergy states [10]. Knowing the quasienergy states of the system one obtains the solution of the Schrödinger equation as a sum over these states. It is then obvious that the decay rate of the ground Wannier states is defined by the lifetime of the most stable quasienergy state.

The equation for the quasienergy states has the form

$$
\widehat{U} \psi=\exp \left(-\frac{i}{\hbar} \mathcal{E} T_{\omega}\right) \psi, \quad T_{\omega}=\frac{2 \pi}{\omega},
$$

where $\widehat{U}$ is the system evolution operator over a period of the driving force. Since we are interested in metastable states, Eq. (7) should be accomplished by a nonhermitian resonance-like boundary condition, which ensures that the quasienergy spectrum $\mathcal{E}=E-i \Gamma / 2$ is discrete. In the general case of an arbitrary $\omega$, Eq. (7) is difficult to solve (at least, we are not aware of any method). Fortunately the case of rational $\omega / \omega_{B}$

$$
\frac{\omega}{\omega_{B}}=\frac{r}{q}, \quad \omega_{B}=\frac{\pi M a}{\hbar k_{L}}
$$

is an exclusion. In this case the solution can be found explicitly by using a simple method described in Refs. [5,
11]. In what follows we calculate the quasienergy spectrum for rational $\omega / \omega_{B}$ and interpolate the result for irrational values to find the dependence $\tau(\omega)$ for arbitrary values of the driving frequency.

A general discussion of the structure of the quasienergy spectrum might be useful [11-13]. For rational $\omega / \omega_{B}$ the eigenfunctions of Eq. (7) are Bloch-like functions and can be labeled by the quasimomentum $k$. The quasienergy spectrum, defined in the interval $0 \leq E<\hbar \omega$, consists of a set of bands originating from different Wannier-Stark ladders (which we label by the index $\alpha$ ). Each set consists of $r$ identical bands, where the Brillouin zone is given by the interval $0 \leq k<k_{L} / r$. There is strong numerical evidence (supported by a perturbation analysis [14]) that for small driving amplitude $\epsilon$ the $k$-dependence of the quasienergy band can be well approximated by a cosine dependence. Thus the spectrum is given by the equation

$$
\begin{aligned}
& \qquad E_{\alpha}(k) \approx \bmod _{\hbar \omega}\left\{\bar{E}_{\alpha}+\hbar \omega \frac{n}{r}+\frac{\Delta E_{\alpha}}{2} \cos \left(\frac{\pi k}{r k_{L}}\right)\right\} \\
& \qquad n=0, \ldots, r-1 \\
& \text { for the real part of the quasienergy, and by }
\end{aligned}
$$

$$
\Gamma_{\alpha}(k) \approx \bar{\Gamma}_{\alpha}+\frac{\Delta \Gamma_{\alpha}}{2} \cos \left(\frac{\pi k}{r k_{L}}\right)
$$

for the imaginary part. In Eqs. (9)-(10) the quantities $\bar{E}_{\alpha}$, $\bar{\Gamma}_{\alpha}$, and $\Delta E_{\alpha}, \Delta \Gamma_{\alpha}$ are functions of both the frequency and amplitude of the driving force. The band widths $\Delta E_{\alpha}$, $\Delta \Gamma_{\alpha}$ are given by the values of the integers $r$ and $q$ in condition (8) (but not their ratio!). In this sense the functions $\Delta E_{\alpha}=\Delta E_{\alpha}(\omega, \epsilon)$ and $\Delta \Gamma_{\alpha}=\Delta \Gamma_{\alpha}(\omega, \epsilon)$ are discontinuous functions of the frequency $\omega$. In contrast, as shown below, the functions $\bar{E}_{\alpha}=\bar{E}_{\alpha}(\omega, \epsilon)$ and $\bar{\Gamma}_{\alpha}=\bar{\Gamma}_{\alpha}(\omega, \epsilon)$ are continuous function of the driving frequency. For $\epsilon=0$ the band widths $\Delta E_{\alpha}, \Delta \Gamma_{\alpha}$ vanish and the values of $\bar{E}_{\alpha}$, $\bar{\Gamma}_{\alpha}$ coincide with the position (reduced to the fundamental energy interval $\hbar \omega_{B}$ ) and the width of the $\alpha$-th Wannier resonances.

To avoid any misunderstanding we note the difference between the dispersion relation (9)-(10) and the dispersion relation given in Ref. [13]. In the paper cited the system (1)-(2) was studied on the basis of the tight-binding model, which can be derived from the original Hamiltonian by using the so-called one-band approximation. In this approximation the Wannier resonances appear as stationary states. Then $\bar{\Gamma}_{\alpha}=\Delta \Gamma_{\alpha}=\bar{E}_{\alpha}=0$ and the band width $\Delta E_{0}$ obeys the relation

$$
\Delta E_{0}=(-1)^{q} \Delta_{0} J_{q}\left(\epsilon \frac{\pi r \omega_{B}}{4 \omega_{r e c}}\right),
$$

where $\Delta_{0}$ is the width of the ground Bloch band and $J_{q}(z)$ is the ordinary Bessel function. Equations (9) and (11) were called a "fractional Stark ladder" in Ref. [13] to stress its discontinuous character. Keeping in mind the initial problem, Eq. (11) has a limited validity and can be used only in the limit of a weak static force and for $\epsilon \ll 1[14]$. 


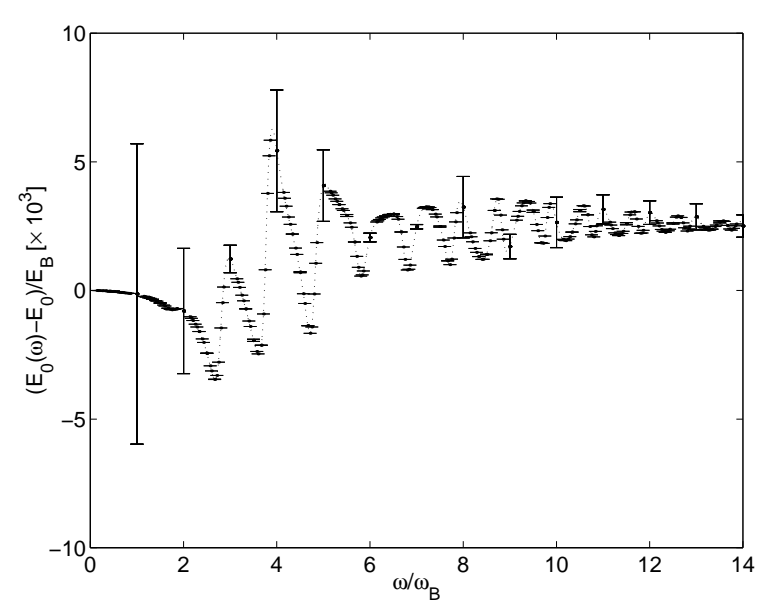

Fig. 1. The position of the ground quasienergy resonance $\bar{E}_{0}$ as a function of the driving frequency $\omega$ for $\hbar=2, F=0.061$, and $\epsilon=0.08$. The dashed line interpolates the values of $\bar{E}_{0}$ obtained for the rational values $\omega / \omega_{B}=r / q, q \leq 7$. The "error bars" correspond to the band width $\Delta E_{0}$. (The case of unresolved band width appears as "minus over dot" sign in the figure.)

4. We proceed with the numerical results. In the calculations we use a scaled coordinate $x^{\prime}=2 k_{L} x$ and time $t^{\prime}=\Omega_{p h} t$, where $\Omega_{p h}=\left(8 \omega_{r e c} V_{0} / \hbar\right)^{1 / 2}$ is the frequency of atomic oscillations near the bottoms of the potential wells ( $\omega_{\text {rec }}=\hbar k_{L}^{2} / 2 M$ is the so-called recoil frequency). Using this scaling the Hamiltonian (1)-(2) takes a dimensionless form (primes are omitted)

$$
\widehat{H}=\hat{p}^{2} / 2+\cos [x-\epsilon \cos (\omega t)]+F x,
$$

where the value of the scaled Planck constant (entering the momentum operator) is $\hbar^{\prime}=\left(8 \hbar \omega_{\text {rec }} / V_{0}\right)^{1 / 2}$, and the static force is $F=M a k_{L}^{-1} / 2 V_{0}$. In what follows we choose $\hbar=2$ (prime is omitted), $F=0.061$, and $\epsilon=0.08$. For these values of $\hbar$ and $F$ the scaled width of the unperturbed ground Wannier resonances is $\Gamma_{0}=1.25 \cdot 10^{-4}$, the first excited $\Gamma_{1}=1.30 \cdot 10^{-1}$, and the distance between the resonances $\bmod _{\hbar \omega_{B}}\left(E_{1}-E_{0}\right)=0.216 \hbar \omega_{B},\left(\omega_{B}=2 \pi F / \hbar\right)$. The dots in Fig. 1 and Fig. 2 show the position $\bar{E}_{0}$ and mean width $\bar{\Gamma}_{0}$ of the most stable quasienergy resonance for "rational" values of the driving frequency $\omega=\omega_{B} r / q$ with $q \leq 7$ and $r \leq 42$. (The quasienergy (9) is normalized by the Bloch quantum $E_{B}=\hbar \omega_{B}$ in Fig. 1 and the quasienergy resonance width (10) is normalized by the energy resonance width of the unperturbed Wannier-Stark states $\Gamma_{0}$ in Fig. 2.) It is seen that the points are arranged along a smooth curve interpolating the required dependence $\tau^{-1}(\omega)=\bar{\Gamma}_{0}(\omega) / \hbar$. In addition, the "error bars" in the figures give the widths $\Delta E_{\alpha}, \Delta \Gamma_{\alpha}$ of the quasienergy bands. We note that, in qualitative agreement with Eq. (11), only the bands corresponding to $q=1$ have a large width in Fig. 1. For $q=2$ the band widths $\Delta E_{0}$ are small and for $q \geq 3$ they are already not resolved on the scale of Fig. 1.

The function $\bar{\Gamma}_{0}(\omega)$ depicted in Fig. 2 is in qualitative agreement with the one expected on the basis of the

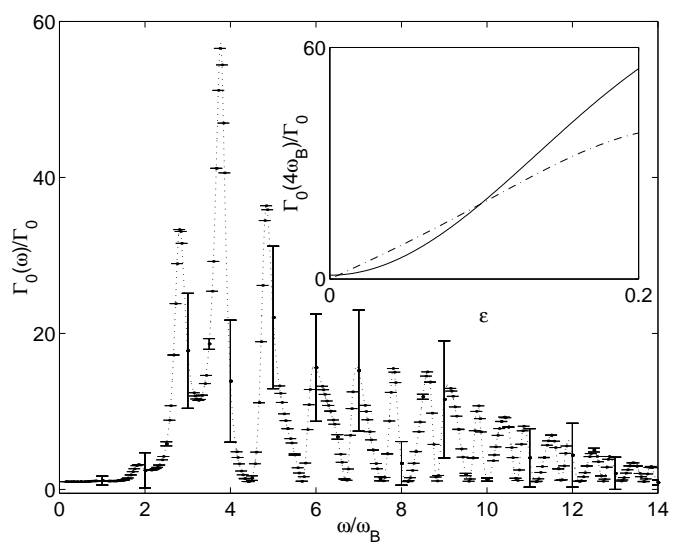

Fig. 2. Same as in Fig. 1 but for the normalized resonance width. The inset shows the mean resonance width $\bar{\Gamma}_{0}=$ $\bar{\Gamma}_{0}(\omega, \epsilon)$ (solid line) and the band width $\Delta \Gamma_{0}=\Delta \Gamma_{0}(\omega, \epsilon)$ (dashed line) as a function of $\epsilon$ for $\omega=4 \omega_{B}$.

Fermi rule. To check the $\epsilon$-dependence of this function we repeated the calculation for a two times smaller value $\epsilon=0.04$. The comparison between the shapes of the curves is given in Fig. 3, where we plot $\bar{\Gamma}_{0}(\omega, \epsilon=0.08) / \Gamma_{0}$ (solid line) and $\left[4\left(\bar{\Gamma}_{0}(\omega, \epsilon=0.04)-\Gamma_{0}\right)+\Gamma_{0}\right] / \Gamma_{0}=4 \bar{\Gamma}_{0}(\omega, \epsilon=$ $0.04) / \Gamma_{0}-3$ (dots). The perfect matching of the dots to the solid line confirms the $\epsilon^{2}$-dependence for the width of the perturbed Wannier resonances. The dashed line in the figure corresponds to function (6), where the transition matrix elements $\left|d_{l}\right|$ are adjusted according to the heights of the peaks. A reasonable coincidence between the curves (together with the $\epsilon^{2}$-dependence) indicates an applicability of the Fermi rule as the simplest approach. However, there are also noticeable deviations from Eq. (6). Further analysis shows that these deviations are due to neglecting the second excited $(\alpha=2)$ Wannier-Stark resonances, which become important for large $\omega\left(\omega>5 \omega_{B}\right.$ for the present choice of the system parameters), and due to neglecting interference effects [15].

Now we briefly discuss the effect of a finite band width $\Delta \Gamma_{0}$ for rational $\omega / \omega_{B}$. First of all we note that, similar to the case of Fig. 1, only the bands corresponding to $\omega=r \omega_{B}$ have non-negligible widths. Moreover, even for these values of $\omega$, the mean $\bar{\Gamma}_{0}$ is typically larger than the band width $\Delta \Gamma_{0}$ (see inset in Fig. 2) and, therefore, the relative effect of the finite band width on the decay process is weak. An exclusion is the case of a very weak driving, where $\Delta \Gamma_{0}$ can be twice as large as $\bar{\Gamma}_{0}$. In this case, as follows from Eq. (10), the decay rate of the quasienergy states corresponding to the edge of the Brillouin zone is smaller than the decay rate of the unperturbed Wannier states. In other words, we encounter a kind of stabilization phenomenon. In the simplest case $r=q=1$ this stabilization by the periodic driving was studied in Ref. [14]. It was shown that for a typical initial condition (almost uniform population of the ground Bloch band, which then adiabatically transforms into the ground Wannier-Stark ladder) the decrease of the population is tremendously 
slowed down and essentially deviates from an exponential decay.

5. Finally we analyze the system (1)-(2) for the parameters of the experiment [8]. These are $\omega_{\text {rec }} / 2 \pi=25.14 k \mathrm{~Hz}$, $V_{0} / h=75 \pm 7 \mathrm{kHz}$ (below we use $V_{0} / h=68 \mathrm{kHz}$ ), $a=$ $1570 \mathrm{~m} / \mathrm{s}^{2}$, which corresponds to a scaled Planck constant $\hbar=1.747$ and a scaled static field $F=0.061$ in the dimensionless Hamiltonian (12), and $\epsilon=0.096$. The dependence $\Gamma_{0}(\omega)$ is depicted in Fig. 4. We note that in view of the perturbation approach the value $\epsilon=0.096$ (for a given value of $\hbar=1.747$ ) should be considered as pretty large. For this $\epsilon$ we find a deviation from the cosine dependence for the dispersion relation of the quasienergy bands $\mathcal{E}_{0}(k)$ and a deviation from the $\epsilon^{2}$-dependence for $\bar{\Gamma}_{0}(\omega)$. In all other aspects the function $\Gamma_{0}(\omega)$ is similar to that presented in Fig. 2. Using the obtained dependence $\bar{\Gamma}_{0}(\omega)$ (the band widths can be neglected) we calculated the survival probability $P_{t}(\omega)=\exp \left[-\bar{\Gamma}_{0}(\omega) t / \hbar\right]$ for $t=170 \mu \mathrm{s}$ which corresponds to the observation time in the experiment (see the inset in Fig. 4). It is seen that the obtained dependence $P_{t}(\omega)$ fits well to the experimental data.

To conclude, we presented a method of calculating the induced decay rate of the ground Wannier states affected by an ac field. This method was used to calculate the survival probability measured in the experiment [8] and a nice coincidence between theory and experiment was noticed. We also would like to note that the discussed laboratory experiment suggests (besides a possibility of measuring $\left.\omega_{B}\right)$ a unique possibility of measuring the width $\Gamma_{1}$ of the resonances belonging to the first excited Wannier-Stark ladder. In fact, inverting the survival probability back to $\bar{\Gamma}_{0}(\omega)$ one finds $\Gamma_{1}$ as the width of the peaks in the dependence $\bar{\Gamma}_{0}(\omega)$.

\section{References}

[*] Also at L. V. Kirensky Institute of Physics, 660036 Krasnoyarsk, Russia.

1. F. Bloch, Z. Phys. 52, (1929) 555 .

2. G. Zener, Proc. R. Soc. London, Ser. A 137, 523 (1934); L. D. Landau, Phys. Z. Sov. 1, 46 (1932);

3. G. H. Wannier, Phys. Rev. 117, 432 (1960).

4. J. Zak, Phys. Rev. Lett. 20, 1477 (1966).

5. M. Glück, A. R. Kolovsky, H. J. Korsch, Phys. Rev. Lett. 82, 1534 (1999); Phys. Rev. E 60, 247 (1999).

6. R. W. Koss and L. M. Lambert, Phys. Rev. B 51479 (1972).

7. For a review, see E. E. Mendez and G. Bastard, Phys. Today 46, No. 6, (1993) 34; F. Rossi, Semicond. Sci. Technol. 13, 147 (1998); K. Leo, Semicond. Sci. Technol. 13, 249 (1998).

8. S. R. Wilkinson, C. F. Bharucha, K. W. Madison, Qian Niu, and M. G. Raizen, Phys. Rev. Lett. 76, 4512 (1996); see also K. W. Madison, M. C. Fisher, and M. G. Raizen, Phys. Rev. A 60, R1767 (1999), for recent progress in the experiment.

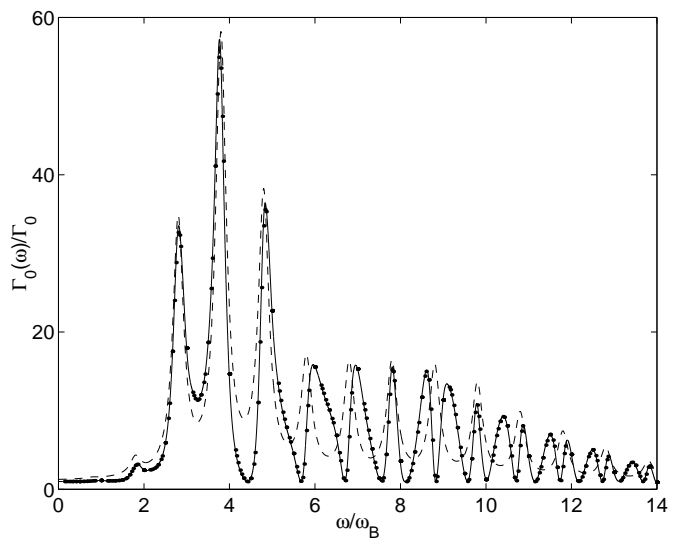

Fig. 3. Comparison between the shape of the function $\bar{\Gamma}_{0}(\omega)$ given in Fig. 2 (solid line) with that for $\epsilon=0.04$ (dots). The dashed line is the function (6), where the coefficients $\left|d_{l}\right|$ are adjusted according to the peaks heights.

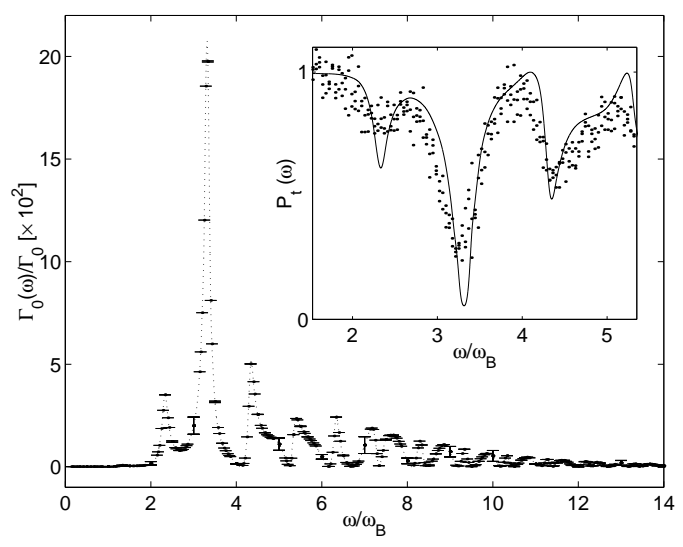

Fig. 4. The same as in Fig. 2 but for the system parameters used in the experiment [8]. The solid line in the insert shows the survival probability $P_{t}(\omega)=\exp \left[-\bar{\Gamma}_{0}(\omega) t / \hbar\right]$ for $t=170 \mu s$. The dots are the experimental data.

9. Usually the Fermi rule refers to a quantum system affected by ac field. It is easy to show that this is actually the case of the system (1)-(2), because the Hamiltonian $\widehat{H}=\hat{p}^{2} / 2 M+$ $V_{0} \cos \left[2 k_{L} x-\epsilon \cos (\omega t)\right]+M a x$ is unitary equivalent to the Hamiltonian $\widehat{H}=\hat{p}^{2} / 2 M+V_{0} \cos \left(2 k_{L} x\right)+M a x+$ $\left(\epsilon \omega^{2} M / 2 k_{L}\right) x \cos (\omega t)$.

10. Ya. B. Zel'dovich, Sov. Phys. JETP 24, 1006 (1967);

11. M. Glück, A. R. Kolovsky, H. J. Korsch, and N. Moiseyev, EPJ D4, 239 (1998);

12. J. Zak, Phys. Rev. Lett. 71, 2623 (1993).

13. X.-G. Zhao, R. Jahnke, and Q. Niu, Phys. Lett. A 202, 297 (1995).

14. M. Glück, A. R. Kolovsky, and H. J. Korsch, Phys. Lett. A 258, 383 (1999).

15. M. Glück, M. Hankel, A. R. Kolovsky, and H. J. Korsch, unpublished. 